\title{
Complications Following Surgical Correction of Medial Patellar Luxation in Small-to-Medium-Size Dogs
}

\author{
Matteo Rossanese ${ }^{1}$ Alexander J. German ${ }^{1,2}$ Eithne Comerford ${ }^{1,2}$ Rob Pettitt ${ }^{1}$ Andrew Tomlinson ${ }^{1}$ \\ Felipe de Vicente ${ }^{3,4}$
}

1 Small Animal Teaching Hospital, School of Veterinary Science, University of Liverpool, Leahurst Neston, United Kingdom

2 Institute of Ageing and Chronic Disease, William Henry Duncan Building, Liverpool, United Kingdom

3 Pride Veterinary Centre, Derby, United Kingdom

${ }^{4}$ Hospital Veterinario Puchol, Madrid, Spain

Vet Comp Orthop Traumatol 2019;32:332-340.

\begin{abstract}
Address for correspondence Matteo Rossanese, DVM, SPSA, CertAVP, MSc, Small Animal Teaching Hospital, School of Veterinary Science, University of Liverpool, Chester High Road, Neston, CH64 7TE, United Kingdom (e-mail: matteo.rossanese@gmail.com).
\end{abstract}

\begin{abstract}
Keywords

- medial patellar luxation

- tibial tuberosity transposition

- trochlear block recession

- trochlear wedge recession

- tension band wire

Objective The main aim of this study was to describe complications in dogs weighing $<20 \mathrm{~kg}$ treated for medial patellar luxation and to determine risk factors associated with these complications.

Materials and Methods Medical records were reviewed and cases were grouped based on four techniques of stabilization for the tibial tuberosity transposition (TTT). Variables including animal signalment, clinical presentation, TTT technique, other concurrent surgical techniques and associated complications were investigated. Multiple logistic regression was used to determine the association of the same variables with specific complications such as tibial tuberosity fractures or avulsions, patellar reluxation and implant-related complications.

Results Of the 100 procedures performed, 37 suffered a complication. There were no significant differences in the overall complication rate between TTT stabilization techniques. Preservation of the tibial tuberosity distal cortical attachment was associated with a reduced risk of implant-related complications, whereas adding a medial release to the surgical techniques was associated with an increased risk of medial patellar reluxation. The type of trochleoplasty performed did not affect the complication rate.

Conclusions In the current study, a high rate of complications was found in dogs $<20 \mathrm{~kg}$ undergoing medial patellar luxation surgery. Performing a medial release and preserving the distal cortical attachment of the tibial tuberosity were the only variables associated respectively with an increase and a reduction in the risk of postsurgical complications.
\end{abstract}

\section{Introduction}

Medial patellar luxation is a common cause of pelvic limb lameness in dogs, particularly in small breeds, with 52 to $65 \%$ being affected bilaterally. ${ }^{1-5}$ Although the exact cause of patellar luxation is unclear, this condition is considered to be developmental, resulting from multiple anatomical abnormalities of the pelvic limbs. ${ }^{4-8}$ Patients with clinical signs associated with medial patellar luxation can be treated surgically with repair techniques that are specifically indicated in cases classified as grades II to IV. ${ }^{9}$ The aim of surgery is to realign the stifle extensor (quadriceps) mechanisms and to reposition the patella within the trochlear groove. Corrective surgical techniques have been investigated and the outcomes have been reported in several studies. ${ }^{8-10}$ The most common surgical technique to realign the quadriceps mechanism is a tibial tuberosity transposition (TTT), which is received

July 24, 2018

accepted after revision

February 6, 2019 (c) 2019 Georg Thieme Verlag KG Stuttgart · New York
DOI https://doi.org/

10.1055/s-0039-1683872. ISSN 0932-0814. 
often combined with a femoral trochleoplasty, lateral soft tissue imbrication and medial soft tissue release. ${ }^{10}$ However, there are individual variations in these techniques, such as the way in which the trochleoplasty is performed and the way in which the tibial osteotomy is stabilized.

The overall complication rate following stabilization of unilateral patellar luxation has been reported as 13 to $45 \%$ including all the surgical correction for different medial patellar luxation grades. ${ }^{1,8-13}$

Complications include patellar reluxation, implant failure or migration, tibial tuberosity fracture and tibial tuberosity avulsion, recession wedge displacement, tibial or femoral fracture, lateral trochlear ridge fracture, patellar ligament rupture, inability to fully extend the stifle joint, osteomyelitis, wound dehiscence and septic arthritis. ${ }^{1,8-13}$

The primary aim of this study was to determine if the method of trochlear recession (block vs. wedge) and the implant construct ( 1 pin vs. 2 pin and tension band wire [TBW] vs. no TBW) used to stabilize the TTT influenced the complication rate of patients weighing $<20 \mathrm{~kg}$ treated for medial patellar luxation. In light of our clinical experience, we hypothesized that the type of surgical technique performed in dogs weighing $<20 \mathrm{~kg}$ would not affect the incidence of complications. Another aim was to report short- and long-term complications ( $>12$ months) for our population and to determine risk factors associated with these complications.

\section{Materials and Methods}

This retrospective observational study used anonymized clinical data and was approved by the Liverpool Veterinary School Research Ethics Committee (VREC470). Patient records were searched to identify dogs $(<20 \mathrm{~kg})$ that underwent surgery for medial patellar luxation between 2011 and 2016.

Dogs were excluded from the study if they had a suspected traumatic patellar luxation, had concurrent orthopaedic or neurological abnormalities (such as cranial cruciate ligament rupture), had previous surgery on either pelvic limb, or if the surgical stabilization was performed with techniques other than trochleoplasty, TTT and soft tissue imbrication or release. Dogs that were re-presented during the study period for treatment of a contralateral medial patellar luxation were included in the study.

Information retrieved from the records included signalment, orthopaedic examination findings, pre- and postoperative radiographic or computed tomographic findings, type of surgical treatment, surgical and anaesthetic time, intraoperative and postoperative complications and need for further surgical or medical treatment. The grade of patellar luxation was recorded according to the Singleton grading system. ${ }^{14}$ Patients were re-examined $\sim 8$ weeks following surgery to evaluate clinical progression and repeat orthogonal radiographs. Patients that exhibited clinical deterioration in the first 8 weeks following surgery were examined sooner and were also examined at any point afterwards if there was any deterioration. Long-term follow-up to detect unreported complications was evaluated using the referring veterinarian clinical notes with a minimum postsurgical time of 12 months.

\section{Surgical Treatment}

Technique combinations performed to achieve intraoperative stability of the patella included medial soft tissue release (medial capsule and retinaculum), lateral capsule and retinaculum imbrication, trochleoplasty and TTT.

All surgical procedures included a lateral parapatellar approach to the stifle joint and an arthrotomy to evaluate the femoral trochlear groove, patellar articular cartilage, cruciate ligaments and menisci. If the femoral trochlea was subjectively considered to be of inadequate depth, a trochlear wedge recession ${ }^{15}$ or a trochlear block recession technique ${ }^{16}$ was performed based on surgeon preference. A TTT was performed if the tibial tuberosity was found to be medially translated, transposing the tibial tuberosity laterally until the quadriceps mechanism was subjectively realigned and the patella could no longer be luxated. The TTT was then stabilized with one or two Kirschner wires (K-wire) with or without a TBW. The K-wires ranged from 0.9 to $2.0 \mathrm{~mm}$, were usually unthreaded and had a trocar point in each end. The point of insertion of the K-wire was recorded as proximal to the tibial tuberosity, at the level of the tibial tuberosity (patellar ligament insertion area) or distal to the tibial tuberosity (or at the tibial crest). When two K-wires were placed, the insertion point was recorded for the most proximal pin, and their location, relative to each other, was described as being vertical or horizontal ( - Fig. 1), as previously described. ${ }^{9}$ The direction of the K-wire was also recorded as caudoproximal, caudodistal or perpendicular to the anatomical axis of the tibia. Soft tissue balancing procedures, including medial release and lateral imbrication, were performed as deemed necessary by the surgeon to ensure normal tracking and stability of the patella throughout stifle range of motion. Orthogonal radiographs were obtained immediately after surgery to assess implant positioning.

\section{Complications}

Orthopaedic and radiographic assessment was completed at 6 to 8 weeks post-operatively or sooner if any deterioration occurred. When a complication occurred, the patient was treated medically or surgically, having subsequent periodic follow-up appointments until the complication was considered resolved. Complications were standardized as previously defined. ${ }^{17}$ To detect any unreported short- or longterm complications associated with the surgery, the referring veterinarians were contacted, and the complete clinical notes of each patient were reviewed.

\section{Statistical Analysis}

\section{Sample Size Estimation}

A power calculation was used to determine the number of surgeries required to detect a difference in complication rate amongst different surgical procedures. Assuming an overall complication rate of $40 \%, 9,12$ and an $\alpha$ of 0.05 , it was estimated that a total of 98 surgeries would be required for an $80 \%$ power to demonstrate a $25 \%$ difference (i.e. $25 \%$ difference between the means of the groups) in complication rate between methods. 

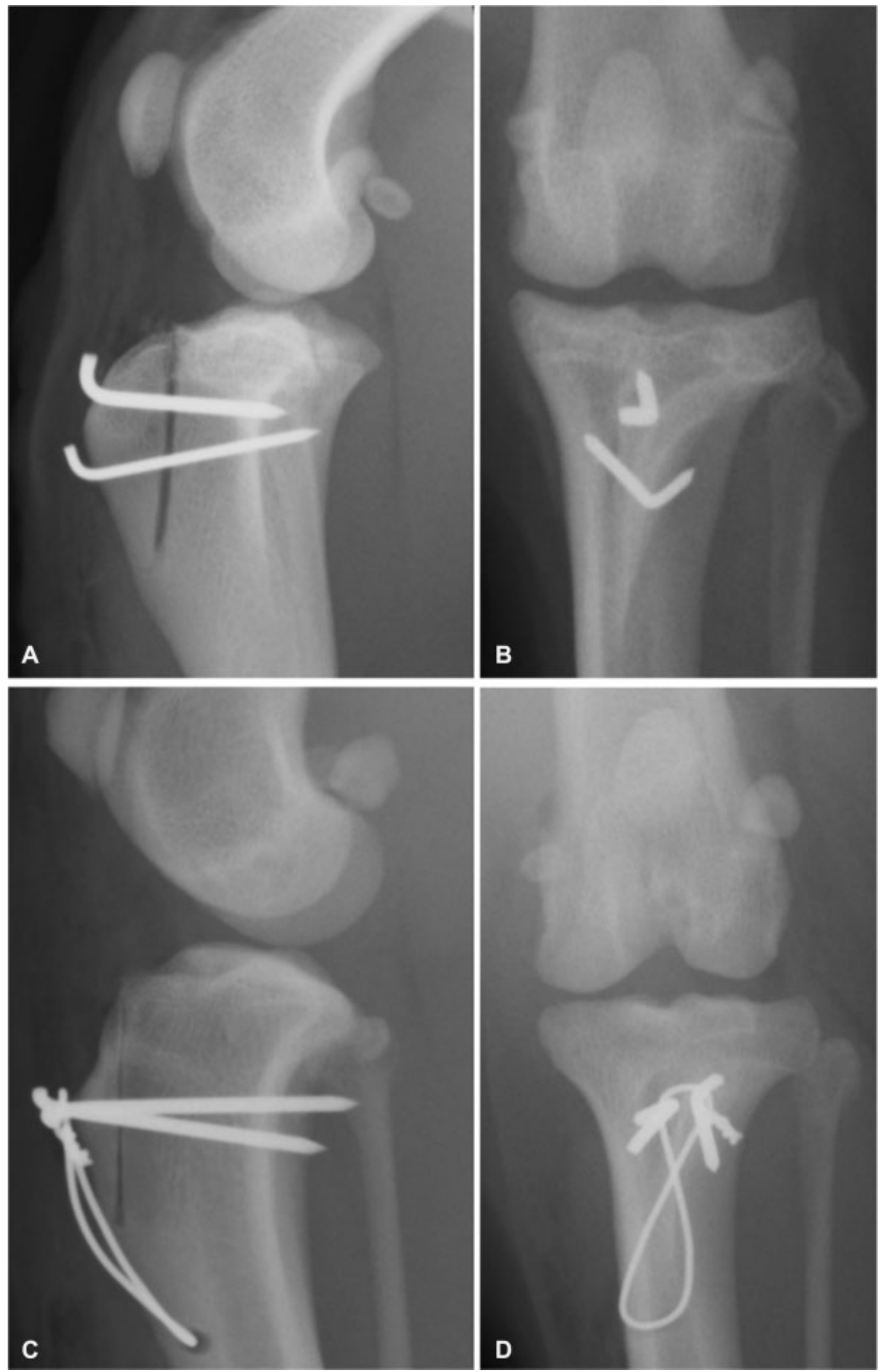

Fig. 1 Mediolateral and caudocranial postoperative radiographs of the stifle showing the position of the two Kirschner wires, relative to the most proximal one, as ( $A$ and $B$ ) vertical or ( $C$ and $D$ ) horizontal.

\section{Initial Statistical Comparisons}

Initially, Fisher's exact test was used to compare differences in the number of complications with different case presentations and surgical methods. Presence or absence of a complication was the dependent variable. Continuous explanatory variables assessed included were age, weight, anaesthetic time and surgical time. Using a Shapiro-Wilk test, none of these datasets were likely to be from a normal distribution $(p<0.001$ for all) and, therefore, they are reported as median (range). Categorical variables assessed were sex, neuter status, breed, presurgical medial patellar luxation grade, use of trochleoplasty, type of trochleoplasty (trochlear wedge recession vs trochlear block recession), use of medial release, use of lateral imbrication, use of TBW, number of K-wires, K-wire point of insertion, 
K-wire direction, TTT distal cortical attachment, surgeon performing the procedure (senior surgeon vs. resident under supervision), K-wire alignment (only when two pins were used) and the use of postoperative antibacterial therapy. With regard to the four different constructs (1 pin, 1 pin + TBW, 2 pins, 2 pins + TBW), Fisher's exact test was initially used. Since there was no difference between the four groups, another analysis was performed taking into account the single variable (number of pins and $+/$ - use of TBW). Finally, the Fisher's exact test was used to determine whether TBW was more likely to be used in cases without an intact distal tibial tuberosity attachment; further, the bodyweight of dogs that did or did not have a TBW was compared with determine whether the use of this procedure differed according to dog size.

\section{Determining Factors Associated with the Risk of Complications}

Simple and multiple logistic regression were used to determine the association of a range of variables with the occurrence of complications. Initially, the outcome variable was occurrence of any type of complication, and the explanatory variables were those listed above for Fisher's exact test. These variables were first tested separately with simple logistic regression. In addition to assessing both bodyweight and the use of TBW separately in simple logistic regression, the interaction between these factors was also tested. Similarly, the interaction between use of a TBW and whether or not the distal tuberosity attachment remained intact was also tested. A multiple logistic model was then built, which initially included the variables identified as $p<0.2$ by simple regression. Contingency tables were used to assess the independence of each factor vis-à-vis other factors. The model was then refined over multiple rounds, using backward-stepwise elimination of the least significant variable each time, and variables were only retained in the final model if they were significant in their own right $(p<0.05)$. To characterize further the variables associated with complications, multiple regression analysis was repeated separately for different types of complications: tibial tuberosity fracture or avulsions, patella reluxation and implant-related complications (including seroma, skin irritation or any cause of implant removal, e.g. pin migration or patella tendinosis). Logistic regression results are reported as odds ratios, 95\% confidence intervals and the associated $p$ value. The level of statistical significance was set at $p<0.05$ for two-sided analyses.

\section{Results}

In total, 87 dogs met the eligibility criteria for the study and a total of 100 surgical procedures for medial patellar luxation were performed. There were 35 male ( 23 neutered) and 52 female dogs ( 34 neutered). At the time of the surgery, the median age was 20 months (range, 3-96 months) and median weight was $8.2 \mathrm{~kg}$ (range, $1.2-20.0 \mathrm{~kg}$ ). The most common represented breeds included Cavalier King Charles Spaniel $(n=17)$, Chihuahua $(n=9)$, Yorkshire Terrier $(n=9)$, French Bulldog $(n=5)$ Jack Russell Terrier $(n=4)$, Bichon Frise $(n=3)$, Border Collie $(n=3)$, Griffon Bruxellois $(n=3)$,
Pomeranian $(n=3)$, Staffordshire Bull Terrier $(n=3)$ and Toy Poodle $(n=3)$. Preoperative imaging aimed to characterize the deformities present in each individual case, and consisted of orthogonal radiographs of femurs, tibias and stifles or computed tomography of both pelvic limbs, or a combination of both imaging modalities. In our study population, corrective osteotomy techniques of the distal femur or proximal tibia were not deemed necessary based on these preoperative imaging studies. Surgery was performed on the left stifle in 60 cases and on the right stifle in 40 cases. The luxation grade was classified as grade I in 1 stifle, grade II in 51 stifles, grade III in 42 stifles and grade IV in 6 stifles.

\section{Surgical Procedures}

Median surgical time was 75 minutes (range, 25-195 minutes) and median anaesthetic time was 135 minutes (range, 50-270 minutes). Surgery was performed by an experienced surgeon in 70 cases and by a resident under supervision in 30 cases. Thirteen dogs had bilateral medial patellar luxation surgery at least 8 weeks apart. Femoral trochleoplasty was performed on 90 stifles and included a trochlear wedge recession in 68 stifles and a trochlear block recession in 22 stifles. Medial soft tissue release was performed on 41 stifles and lateral imbrication was performed in 81 stifles. All surgical procedures included a lateral TTT. The tibial tuberosity was stabilized with one K-wire (range, $0.9-1.8 \mathrm{~mm}$ ) in 28 stifles, one K-wire (range, 0.9-1.6 mm) and TBW (range, $0.8-1.1 \mathrm{~mm}$ ) in 26 stifles, two K-wires (range, 1.1-1.6 mm) in 20 stifles and two K-wires (range, 0.9-1.6 mm) and TBW (range, $0.8-1.6 \mathrm{~mm}$ ) in 26 stifles (-Fig. 2). There was no difference in the bodyweight of dogs that had a TBW compared with those that did not $(p=0.074)$.

The insertion of the K-wire was proximal to the tibial tuberosity in 20 cases (20\%), at the level of the tibial tuberosity in 58 cases (58\%), distal to the tibial tuberosity in 18 cases (18\%) and not assessable in 4 cases (4\%). The direction of the pins was caudodistal in 24 cases (24\%), caudoproximal in 17 cases (17\%) and perpendicular to the long axis of the tibia in the remaining 59 cases (59\%) (-Fig. 3). When two K-wires were used (46 cases), the alignment was vertical in 34 cases and horizontal in 12 cases (-Fig. 1). On the postoperative radiographs, the distal cortical attachment of the tibial tuberosity was deemed to be preserved in 30 stifles, not maintained in 43 stifles and could not be assessed in 27 stifles due to rotation. There was no difference in use of TBWs for cases where the distal attachment of the tibial tuberosity was or was not left intact $(p=1.000)$. Antibacterial therapy was prescribed postoperatively at the discretion of the surgeon, and 16 stifles received antibiotic treatment for 5 to 7 days postoperatively.

\section{Complications}

Thirty-seven stifle joints developed postoperative complications; 12 were considered minor and 25 were considered major. No catastrophic complications were reported (-Table 1). Twenty-eight complications (12 minor and 18 major) occurred in the perioperative period and 7 ( 7 major) occurred between 3 and 6 months after surgery. No mid- 

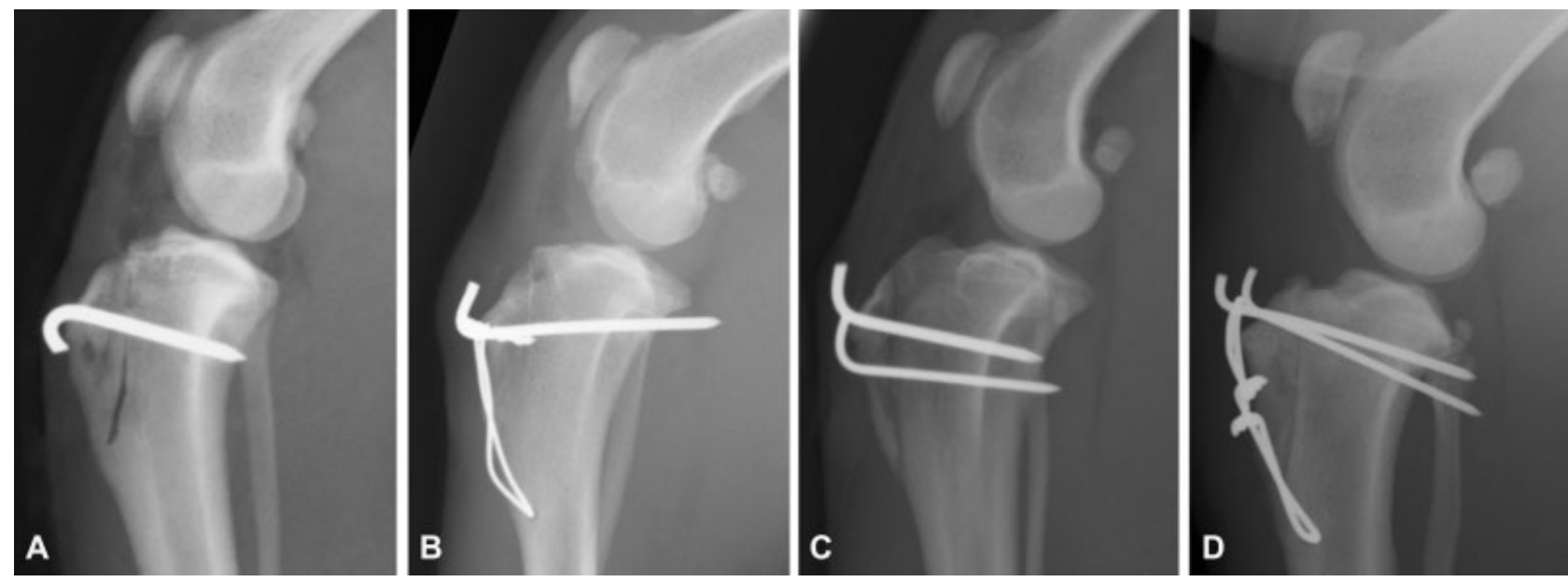

Fig. 2 Mediolateral postoperative radiographs of the stifle showing the four different techniques: (A) one K-wire, (B) one K-wire with tension band wire (TBW), (C) two K-wires and (D) two K-wires with TBW.
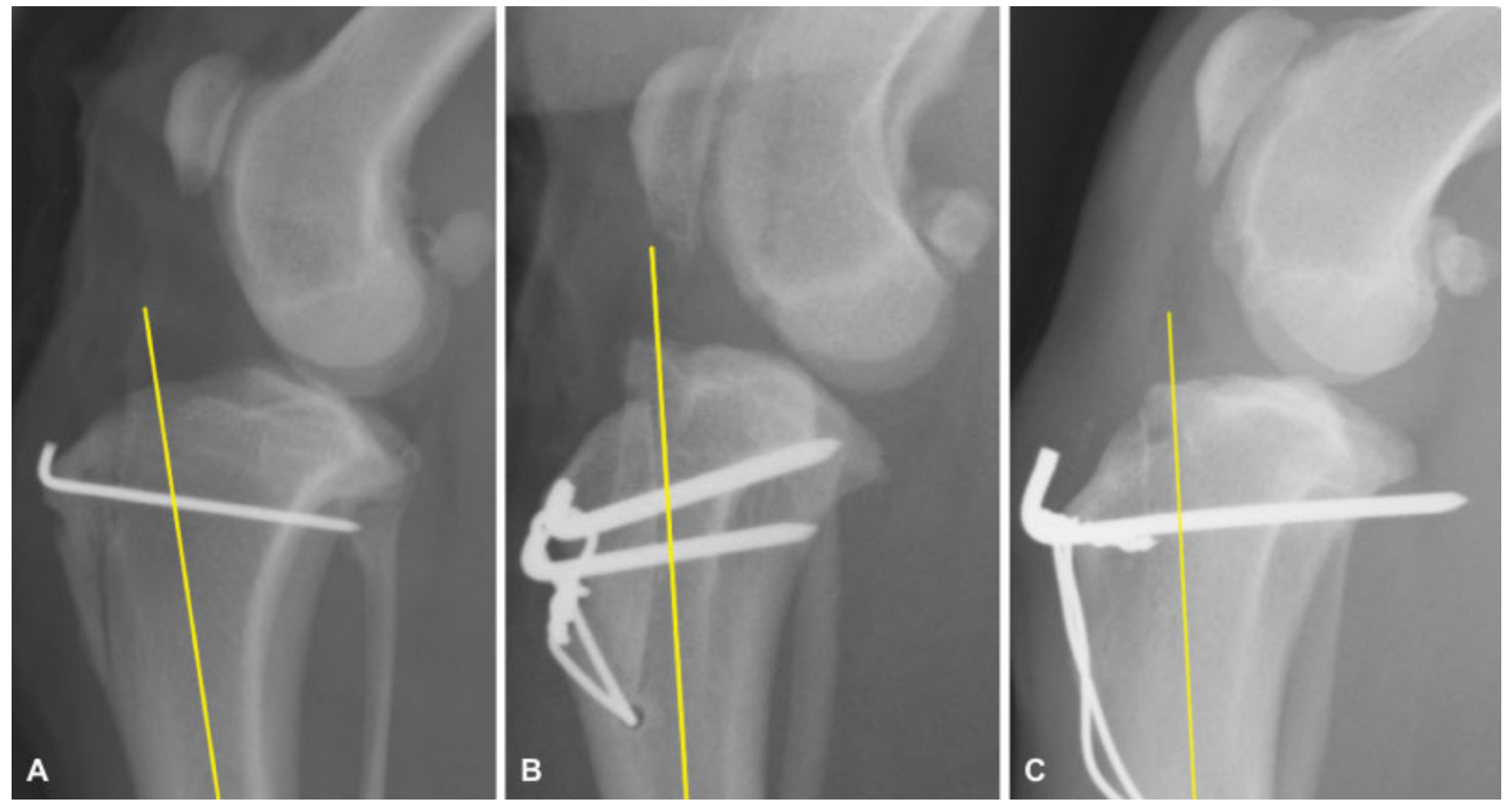

Fig. 3 Mediolateral postoperative radiographs of the stifle showing the direction of the K-wire in relation to the anatomical axis of the tibia: (A) caudodistal, (B) caudoproximal (C) and perpendicular.

term or long-term complications were reported. Postoperative follow-up ranged from a minimum of 15 months to a maximum of 61 months (median 27 months).

When the TTT was stabilized with a single K-wire, complications occurred in 9/28 cases, with 6 of these requiring a second surgery either to remove the K-wire due to skin irritation $(n=2)$, infection $(n=1)$ or pin migration $(n=2)$, or to treat a tibial tuberosity fracture $(n=1)$. There were four minor complications: three cases of patellar reluxation (grade I) occurred but second surgery was not considered necessary, and one tibial tuberosity fracture which was an incidental finding on follow-up radiographs and did not require any specific treatment. One patient had two concurrent complications, with one being classified as major (surgical site infection) and the other as minor (grade I patellar reluxation).

When the TTT was stabilized with two K-wires, complications occurred in $6 / 20$ cases. The complications were classified as major in four cases and minor in two cases. Major complications involved removal of the K-wires due to skin irritation $(n=2)$ or seroma formation $(n=2)$, and minor complications consisted of two tibial tuberosity fractures that did not require further surgical or medical intervention.

When the TTT was stabilized with one K-wire and a TBW, complications occurred in $13 / 26$ cases, with 9 of these requiring a second surgery. There were five implant removals, one femoral growth plate fracture, one patellar reluxation and two tibial tuberosity fractures. The implant 
Table 1 Summary of postoperative major and minor complications following corrective surgery for medial patellar luxation in dogs

\begin{tabular}{|c|c|c|c|c|c|}
\hline \multicolumn{6}{|c|}{ Major complications } \\
\hline Group & Implant related $^{\mathrm{a}}$ & Reluxation & TTF/Avulsion & Others & Total \\
\hline $1 \mathrm{~K}$-wire & 4 & & 1 & 1 (SSI) & $6 / 28$ \\
\hline $1 \mathrm{~K}$-wire + TBW & 5 & 1 & 2 & 1 (femoral fracture) & $9 / 26$ \\
\hline 2 K-wire & 4 & & & & $4 / 20$ \\
\hline 2 K-wire + TBW & 4 & 1 & & 1 (tibial fracture) & $6 / 26$ \\
\hline \multicolumn{6}{|c|}{ Minor complications } \\
\hline $1 \mathrm{~K}$-wire & & 3 & 1 & & $4 / 28$ \\
\hline $1 \mathrm{~K}$-wire + TBW & 3 & & & 1 (wound dehiscence) & $4 / 26$ \\
\hline 2 K-wire & & & 2 & & $2 / 20$ \\
\hline 2 K-wire + TBW & 1 & 1 & & & $2 / 26$ \\
\hline
\end{tabular}

Abbreviations: SSI, surgical site infection; TBW, tension band wire.

amplant-related complications included seroma, skin irritation, or any cause of implant removal, for example, pin migration or patellar desmitis.

removals were performed due to skin irritation $(n=3)$ and pin displacement $(n=2)$. Four minor complications occurred including seroma formation $(n=2)$, minimal wound dehiscence $(n=1)$ and minor open wound due to pin irritation $(n=1)$.

When the TTT was stabilized with two K-wires and TBW complications occurred in 8/26 cases, 6 of which were major and 2 were minor. Major complications included four implant removals, one tibial fracture and one patellar reluxation (grade II). Implant removals were caused by patellar ligament desmitis $(n=1)$, pin migration $(n=2)$ and seroma $(n=1)$; minor complications included seroma formation $(n=1)$ and patellar reluxation $(n=1$, grade II).

Comparisons were made among the four construct groups (one pin, one pin-TBW, two pins, two pins-TBW). There was no statistical difference in the incidence of complications between the four different groups as separate categories ( $p=0.850$ ) or comparing them after grouping them by the use of one-two pins ( $p=0.834$ ), or by the use or not of a TBW $(p=0.560)$. There was no difference in the proportion.

\section{Risk Factors Associated with Complications of Surgery for Patellar Luxation}

Logistic regression analysis was used to determine factors associated with the occurrence of complications, when taking into account possible confounding factors (-Table 2). There were no significant interactions between TBW use and either bodyweight ( $p=0.222$ ) or whether or not the distal tibial tuberosity remained attached $(p=0.505)$. After the initial model was refined by backward-stepwise elimination, the best-fit model was one that included four variables. In the final multiple regression model (-Table 3 ), the only factor positively associated with a reduced risk of complications was preserving the TTT distal cortical attachment, whereas factors associated with an increased risk of complications included medial release and caudoproximal pin direction. Finally, these variables were further investigated to determine associations with specific types of complication ( - Table 4). Medial release was associated with an increased risk of medial reluxation after medial patellar luxation, while preserving the distal cortical attachment was associated with a decreased risk of implant-related complications. Pin direction was not associated with any specific type of complication.

\section{Discussion}

This study has demonstrated that the constructs used to stabilize the TTT ( 1 pin, 2 pins, 1 pin with TBW, 2 pins with TBW) and the type of trochleoplasty (wedge vs block) do not affect the occurrence of postoperative complications following medial patellar luxation surgery in dogs weighing $<20 \mathrm{~kg}$. Among the variables analysed, preservation of the distal TTT cortical attachment was associated with a reduction in the postsurgical complication rate, whereas performing a medial soft tissue release was associated with an increase in the postsurgical complication rate.

The $37 \%$ overall complication rate reported in this study is higher than recently reported but consistent with historical data. ${ }^{8-13}$ However, results from different studies should be interpreted cautiously, as there are important differences between them regarding case selection, length of follow-up, surgical techniques, categorization of minor and major complications, presence of concurrent orthopaedic pathology and comparison between unilateral, bilateral staged and bilateral single session surgical procedure. Implant-related complications requiring a second surgery represented the most frequent complication in our study $(n=17)$. The reason for this is unknown but the authors speculate that this might be due to the relatively small soft tissue coverage around the surgical site which could potentially increase the risk of skin irritation or seroma formation. Another explanation is that in some cases an ongoing instability is likely to have contributed to pin migration and, therefore, led to implant removal.

Patellar reluxation is a commonly reported complication and this finding is supported by our study $(n=6)$. Reasons for patella reluxation can include an inadequate degree of TTT, concurrent femoral or tibial angular limb deformities or a postsurgical inadequate trochlear groove depth. It has been 
Table 2 Simple logistic regression results determining factors associated with complications after surgical correction of medial patellar luxation in dogs

\begin{tabular}{|c|c|c|c|}
\hline \multirow[t]{2}{*}{ Logistic regression } & \multicolumn{3}{|c|}{ Complications } \\
\hline & OR & $95 \% \mathrm{Cl}^{\mathrm{a}}$ & $p$-Value \\
\hline Age & 0.99 & $0.98-1.01$ & 0.966 \\
\hline Gender & 1.46 & $0.63-3.40$ & 0.374 \\
\hline Neuter status & 2.50 & $1.03-6.05$ & 0.042 \\
\hline Body weight & 1.01 & $0.93-1.10$ & 0.680 \\
\hline Surgeon's experience & 0.73 & $0.30-1.77$ & 0.493 \\
\hline Surgical time & 0.99 & $0.98-1.01$ & 0.794 \\
\hline Anaesthetic time & 0.99 & $0.98-1.01$ & 0.836 \\
\hline Trochleoplasty & 0.31 & $0.08-1.21$ & 0.092 \\
\hline TWR & 1.27 & $0.52-3.13$ & 0.590 \\
\hline TBR & 0.33 & $0.10-1.09$ & 0.069 \\
\hline Medial release & 1.93 & $0.83-4.45$ & 0.121 \\
\hline Lateral imbrication & 0.59 & $0.20-1.67$ & 0.321 \\
\hline \multicolumn{4}{|l|}{ Constructs } \\
\hline 1 pin & 0.83 & $0.33-2.11$ & 0.708 \\
\hline 2 pins & 0.75 & $0.26-2.17$ & 0.600 \\
\hline TBW & 1.37 & $0.60-3.14$ & 0.450 \\
\hline $\begin{array}{l}\text { Distal tuberosity } \\
\text { attachment }\end{array}$ & 0.17 & $0.05-0.59$ & 0.005 \\
\hline \multicolumn{4}{|l|}{ MPL grade } \\
\hline Grade I & Ref & & \\
\hline Grade II & 1.17 & - & 0.997 \\
\hline Grade III & 1.30 & - & 0.997 \\
\hline Grade IV & 1.17 & - & 0.997 \\
\hline \multicolumn{4}{|l|}{ K-wire insertion } \\
\hline $\begin{array}{l}\text { Proximal to the } \\
\text { tibial tuberosity }\end{array}$ & Ref & & \\
\hline At the tibial tuberosity & 0.45 & $0.16-1.20$ & 0.113 \\
\hline $\begin{array}{l}\text { Distal to the } \\
\text { tibial tuberosity }\end{array}$ & 0.94 & $0.27-3.22$ & 0.928 \\
\hline \multicolumn{4}{|l|}{ K-wire direction } \\
\hline Caudodistal & Ref & & \\
\hline Caudal & 1.34 & $0.48-3.74$ & 0.571 \\
\hline Caudoproximal & 4.92 & $1.56-15.5$ & 0.006 \\
\hline
\end{tabular}

Abbreviations: $\mathrm{Cl}$, confidence interval; MPL, medial patellar luxation; OR, odds ratio; Ref, reference; TBR, trochlear block recession; TBW, tension band wire; TWR, trochlear wedge recession.

${ }^{a}$ 95\% Cl: ninety-five per cent confidence interval: Reference category used in logistic regression. Variables highlighted in bold qualified for inclusion in the multiple regression analysis at $p<0.20$ ( - Table 3 ).

suggested that failure to address a shallow trochlear groove is a risk factor for reluxation, ${ }^{18}$ while dogs that undergo medial patellar luxation surgery without trochleoplasty are five times more likely to develop reluxation. ${ }^{9}$ A trochleoplasty was performed in $90 \%$ of our cases. The type of
Table 3 Multiple logistic regression results determining factors associated with all complications after surgical correction of medial patellar luxation in dogs

\begin{tabular}{|l|c|c|c|}
\hline Logistic regression & \multicolumn{3}{|c|}{ Complications } \\
\hline & OR & $95 \% \mathrm{Cl}$ & $p$-Value \\
\hline $\begin{array}{l}\text { Distal tuberosity } \\
\text { attachment }\end{array}$ & 0.18 & $0.04-0.72$ & 0.015 \\
\hline Medial release & 3.22 & $1.00-10.34$ & 0.048 \\
\hline $\begin{array}{l}\text { Pin direction } \\
\text { (caudoproximal) }\end{array}$ & 4.53 & $1.01-20.36$ & 0.048 \\
\hline
\end{tabular}

Abbreviations: OR, odds ratio; $95 \% \mathrm{Cl}$, ninety-five per cent confidence.

Table 4 Multiple logistic regression results determining factors associated with specific types of complication after surgical correction of medial patellar luxation in dogs

\begin{tabular}{|l|l|l|l|}
\hline Logistic regression & \multicolumn{3}{|c|}{ Complications } \\
\hline & OR & $95 \% \mathrm{Cl}$ & $p$-Value \\
\hline Implant-related & & & \\
\hline $\begin{array}{l}\text { Distal tuberosity } \\
\text { attachment }\end{array}$ & $\mathbf{0 . 1 0}$ & $\mathbf{0 . 0 1 - 0 . 8 2}$ & $\mathbf{0 . 0 3 2}$ \\
\hline Medial release & 2.06 & $0.55-8.10$ & 0.297 \\
\hline Pin direction & 2.54 & $0.52-12.34$ & 0.245 \\
\hline Reluxation & & & \\
\hline $\begin{array}{l}\text { Distal tuberosity } \\
\text { attachment }\end{array}$ & 0.36 & $0.02-5.71$ & 0.475 \\
\hline Medial release & 9.94 & $\mathbf{1 . 1 4 - 8 6 . 0 5}$ & $\mathbf{0 . 0 3 7}$ \\
\hline Pin direction & 2.26 & $0.14-34.41$ & 0.555 \\
\hline TTF/avulsion & & & 0.582 \\
\hline $\begin{array}{l}\text { Distal tuberosity } \\
\text { attachment }\end{array}$ & 0.50 & $0.04-5.64$ & 0.745 \\
\hline Medial release & 1.40 & $0.17-11.24$ & 0.709 \\
\hline Pin direction & 1.62 & $0.12-20.49$ & \\
\hline
\end{tabular}

Abbreviations: OR, odds ratio; $95 \% \mathrm{Cl}$, ninety-five per cent confidence; TTF/avulsion: tibial tuberosity fracture avulsion.

trochleoplasty performed was decided on individual basis by the surgeon. In a cadaveric study comparing both types of trochlear recession, trochlear block recession provided a larger recessed percentage of trochlear surface area with increased patellar articular contact and greater resistance to patellar luxation in a stifle extended position than trochlear wedge recession. ${ }^{19}$ It seems then plausible that patients that have a trochlear wedge recession would have a higher postsurgical reluxation rate. Results from our study showed no significant difference in complication rates between the two techniques. To the authors knowledge, this is the first study comparing clinical outcomes of these two techniques.

Another major complication reported in the current study involved either fracture or avulsion of the tibial tuberosity. ${ }^{6}$ This was observed in $6 \%$ stifles, with three cases requiring additional surgery and three cases being managed conservatively. Tibial tuberosity avulsions have previously been associated with the 
use of a single pin to stabilize the TTT, and with a caudodistal pin insertion angle. ${ }^{6}$ It has also been suggested that the use of a TBW could prevent a tibial tuberosity fracture. ${ }^{19}$ However, our results contradict this in that we did not identify a specific construct for stabilizing the tibial tuberosity associated with an increased risk of postsurgical tibial tuberosity fracture or avulsions. An attempt was made to analyse if the location of pin insertion in relation to the insertion of the patellar ligament would influence the incidence of tibial tuberosity fractures or avulsion by acting as a stress riser. Our results showed that placement of the most proximal pin proximal, distal or at the level of insertion of the patellar ligament did not have any influence in the rate of surgical complications.

Current recommendations on how to stabilize the TTT involve the placement of 1 or $2 \mathrm{~K}$-wires, directed in a distocaudomedial direction and the placement of a TBW. ${ }^{20}$ Although it has been suggested that using a TBW minimizes the risk of implant-related complications (migration or implant failure) and prevent tibial tuberosity fracture, ${ }^{19}$ only about half of our patients had a TBW placed. No significant difference between the four constructs and the occurrence of complication was found, as well as when the three categories of complication rates (risk of tibial tuberosity fracture or avulsions, risk of reluxation and risk of having an implant-associated complications) were assessed. The only risk factors identified that were associated with having a complication were lack of tibial tuberosity distal cortical attachment during TTT, placing the pin in a caudoproximal direction and performing a medial retinacular release.

In the current study, the medial retinacular release was associated with increased odds of any complication, especially having postoperative patellar reluxation. A possible explanation is that medial release is more likely to be performed in higher grades of medial patellar luxation in which it is challenging to reduce the patella into the trochlear grove without this medial release, hence the negative association with complications. Surprisingly, no significant association was found between the preoperative medial patellar luxation grade and any specific type of complication; this might be due to a type II statistical error. Another possibility could be that the quadriceps mechanism would have not been adequately aligned due to inadequate TTT or failure to recognize and correct femoral varus and therefore complication rates would be expected to be higher. Due to the retrospective nature of the study, it was not possible to assess if the degree of lateral TTT was adequate. However, it must be assumed that the surgeon performing the procedure considered the degree of TTT lateralization adequate during the procedure. Regarding the possible presence of a significant femoral varus, preoperative radiographs and/or computed tomographic scans were not consistent with this possibility.

Placing the pin in a caudoproximal direction was associated with increased overall odds of developing a complication. Given the wide-ranging complications observed, the fact that no association was observed with a specific type of complication on multiple regression might be due to a type II statistical error. Therefore, further studies could be considered to explore the complications associated with caudoproximal pin placement.
The findings of the current study also suggest an association between tibial tuberosity distal cortical attachment preservation and reduced odds of complications with medial patellar luxation surgery, and in particular with a reduced risk of implant-related complications. This suggests a possible increased tibial tuberosity instability when the distal attachment is not preserved. Indeed, a previous study suggested that preservation of the distal cortical extension of the osteotomy segment allows a partial transmission of load forces to the tibia rather than relying solely on the implant fixation and moreover, it is also important for preservation of blood supply. ${ }^{21,22}$ Unfortunately in some postoperative radiographs, it was not possible to determine if the distal TTT cortical attachment was intact, and therefore it is possible that the lack of difference between constructs could have been due to an statistical error.

Only patients weighing $<20 \mathrm{~kg}$ were included in this study as body weight has been reported to be risk factor for the development of complications with a cut-off of $20 \mathrm{~kg}$ : dog weighting $>20 \mathrm{~kg}$ had a higher frequency of overall and major complications and reluxation compared with dogs weighing $<20 \mathrm{~kg} .{ }^{10}$

Limitations of this study include its retrospective design, which relied on the use of clinical records. In particular for techniques such as lateral imbrication and medial soft tissue release, many methods and procedure exist. Based on the retrospective nature of the study, it was not possible to determine what particular technique was used in each dog. The measures of clinical outcome of the dogs in this study were not evaluated. Subjective owner assessment using a questionnaire or kinematic analysis would have been helpful to evaluate the functional outcome; however, this was beyond the scope of this study.

We identified long-term complications by reviewing the referring veterinarian clinical records; therefore, it is possible that some complications were not noticed by the referring veterinarian and were missed. Third, there was variability in the corrective procedures used, which was based on the preference of the surgeon, and there was no standardized protocol to compare the four groups. It was not possible to quantify if the TTT performed was sufficient to realign the quadriceps mechanisms or if the trochleoplasty was deep enough to accommodate permanently the patella. Fourth, although the patients were followed for a minimum of 12 months after surgery, it is possible that later-onset complications might be missed. A fifth limitation is study power; although our power analysis suggested that the study was adequately powered for some variables, we cannot guarantee that this study was adequately powered for every single variable investigated.

In conclusion, the results of the current study suggested no differences in complication rates when different implant constructs for TTT stabilization are used during medial patellar luxation surgery in dogs weighing $<20 \mathrm{~kg}$. However, preserving the distal cortical TTT attachment was associated with decreased postsurgical complications rate, while adding a medial capsule or retinaculum release was associated with increased post-surgical complications. Finally, the type of trochleoplasty performed (trochlear block recession vs. trochlear 
wedge recession) was not associated with the occurrence of postoperative complications.

\section{Author Contribution}

Matteo Rossanese and Felipe De Vicente contributed to conception of study, study design, acquisition of data and data analysis and interpretation. Alexander J. German contributed to study design and data analysis and interpretation. Eithne Comerford, Rob Pettitt and Andrew Tomlinson contributed to acquisition of data and data analysis and interpretation. All Authors drafted, revised and approved the submitted manuscript.

\section{Conflict of Interest}

Dr. GERMAN reports grants, personal fees, non-financial support and other from ROYAL CANIN/MARS PETCARE, other from RAW FEEDING VET SOCIETY, non-financial support and other from PURINA, personal fees and nonfinancial support from SCIVAC, non-financial support and other from NORDIC PETFOOD CONFERENCE, personal fees, non-financial support and other from AAFP CONFERENCE, personal fees, non-financial support and other from AUSTRIAN ASSOC SMALL ANIMAL PRACT, personal fees and non-financial support from ICVC MEETING, personal fees and non-financial support from VMX CONFERENCE, personal fees and non-financial support from LONDON VET SHOW, non-financial support and other from ECVIM, non-financial support and other from BSAVA, non-financial support from CABI SYMPOSIUM, personal fees, non-financial support and other from UNIVERSITY OF CAMBRIDGE, non-financial support and other from WSAVA, personal fees, non-financial support and other from EEVC CONGRESS, personal fees, non-financial support and other from ISFM/ICC, personal fees, nonfinancial support and other from SLOVAKIAN VET CONGRESS, rants from ZOETIS, outside the submitted work. His academic post at the University of Liverpool is financially supported by Royal Canin. Other authors do not have any affiliation, financial support or conflict of interest with the technique or products used.

\section{References}

1 Alam MR, Lee JI, Kang HS, et al. Frequency and distribution of patellar luxation in dogs. 134 cases (2000 to 2005). Vet Comp Orthop Traumatol 2007;20(01):59-64

2 Towle HA, Griffon DJ, Thomas MW, Siegel AM, Dunning D, Johnson A. Pre- and postoperative radiographic and computed tomographic evaluation of dogs with medial patellar luxation. Vet Surg 2005;34(03):265-272

3 Remedios AM, Basher AW, Runyon CL, Fries CL. Medial patellar luxation in 16 large dogs. A retrospective study. Vet Surg 1992;21 (01):5-9
4 Hayes AG, Boudrieau RJ, Hungford LL. Frequency and distribution of medial and lateral patellar luxation in dogs: 124 cases (1982-1992). J Am Vet Med Assoc 1994;205(05):716-720

5 Priester WA. Sex, size, and breed as risk factors in canine patellar luxation. J Am Vet Med Assoc 1972;4:633-636

6 Gibbons SE, Macias C, Tonzing MA, Pinchbeck GL, McKee WM. Patellar luxation in 70 large breed dogs. J Small Anim Pract 2006;47(01):3-9

7 Campbell CA, Horstman CL, Mason DR, Evans RB. Severity of patellar luxation and frequency of concomitant cranial cruciate ligament rupture in dogs: 162 cases (2004-2007). J Am Vet Med Assoc 2010;236(08):887-891

8 Gallegos J, Unis M, Roush JK, Agulian L. Postoperative complications and short-term outcome following single-session bilateral corrective surgery for medial patellar luxation in dogs weighing <15. kg: 50 cases (2009-2014). Vet Surg 2016;45(07):887-892

9 Cashmore RG, Havlicek M, Perkins NR, et al. Major complications and risk factors associated with surgical correction of congenital medial patellar luxation in 124 dogs. Vet Comp Orthop Traumatol 2014;27(04):263-270

10 Arthurs GI, Langley-Hobbs SJ. Complications associated with corrective surgery for patellar luxation in 109 dogs. Vet Surg 2006;35(06):559-566

11 Mostafa AA, Griffon DJ, Thomas MW, Constable PD. Proximodistal alignment of the canine patella: radiographic evaluation and association with medial and lateral patellar luxation. Vet Surg 2008;37(03):201-211

12 Linney WR, Hammer DL, Shott S. Surgical treatment of medial patellar luxation without femoral trochlear groove deepening procedures in dogs: 91 cases (1998-2009). J Am Vet Med Assoc 2011;238(09):1168-1172

13 Dunlap AE, Kim SE, Lewis DD, Christopher SA, Pozzi A. Outcomes and complications following surgical correction of grade IV medial patellar luxation in dogs: 24 cases (2008-2014). J Am Vet Med Assoc 2016;249(02):208-213

14 Singleton WB. The surgical correction of stifle deformities in the dog. J Small Anim Pract 1969;10(02):59-69

15 Slocum B, Devine T. Trochlear recession for correction of luxating patella in the dog. J Am Vet Med Assoc 1985;186(04):365-369

16 Talcott KW, Goring RL, de Haan JJ. Rectangular recession trochleoplasty for treatment of patellar luxation in dogs and cats. Vet Comp Orthop Traumatol 2000;13:39-43

17 Cook JL, Evans R, Conzemius MG, et al. Proposed definitions and criteria for reporting time frame, outcome, and complications for clinical orthopedic studies in veterinary medicine. Vet Surg 2010; 39(08):905-908

18 Oshin A. Complications associated with the treatment of patellar luxation. In: Griffon D, Hamaide A, eds. Complications in Small Animal surgery, Vol.1,. Chichester, UK: John Wiley \& Sons, Ltd; 2016:889-896

19 Johnson AL, Probst CW, Decamp CE, et al. Comparison of trochlear block recession and trochlear wedge recession for canine patellar luxation using a cadaver model. Vet Surg 2001;30(02):140-150

20 Kowaleski MP, Boudrieau RJ, Pozzi A. Stifle joint. In: Johnston SA, Tobias KM, eds. Veterinary surgery small animal, Vol. 2. 2nd ed. St. Louis, MO: Elsevier; 2017:906-998

21 Stanke NJ, Stephenson N, Hayashi K. Retrospective risk factor assessment for complication following tibial tuberosity transposition in 137 canine stifles with medial patellar luxation. Can Vet J 2014;55(04):349-356

22 Kanamiya T, Naito M, Ikari N, Hara M. The effect of surgical dissections on blood flow to the tibial tubercle. J Orthop Res $2001 ; 19(01): 113-116$ 